\title{
The Use of Stochastic Processes in Bridge Maintenance Optimization
}

\author{
Bijan Samali, Keith Crews, Khalid Aboura \& Jianchun Li
}

\author{
Centre for Built Infrastructure Research \\ Faculty of Engineering and Information Technology \\ University of Technology Sydney, Australia
}

\begin{abstract}
We introduce an approach for modelling the structural deterioration of components of bridges for maintenance optimization purposes. The Markov chain model is found in the maintenance and repair problems since the early 60's, is introduced to the maintenance of road infrastructure in the 1980's, and is made to drive the current bridge maintenance optimization systems. While this model results into solvable programming problems and provides a solution, there are a number of criticisms associated with it. We highlight the shortfalls of the Markov model for bridge lifetime assessment and promote the use of stochastic processes.
\end{abstract}

\section{INTRODUCTION}

Bridge maintenance optimization was applied during past decades due to the large costs associated with the management of networks of ageing structures. In the United States, more than $70 \%$ of the bridges were built prior to 1935, and a large percentage of the United Kingdom's current bridge stock was built between the late 1950s and early 1970s. In the state of New South Wales, Australia, around $70 \%$ of the operating bridges were built before 1985, with a significant proportion before the 1940 's. With the near completion of most of the road networks and the ageing of bridges, the emphasis shifted to the maintenance and rehabilitation of the existing infrastructure. A concerted effort was made in the 1970's after the collapse of several bridges in the United States in the late 60's [1]. A number of mandates by governments introduced standards and computerized maintenance optimization approaches. These software tools, known as Bridge Management Systems (BMS), consist of formal procedures and methods for gathering and analyzing bridge condition data. The purpose of a BMS is to predict conditions for bridge stocks and estimate maintenance funding. Pontis [2], one of the most widely used systems, was designed and developed at the request of the US Federal Highway Administration. A similar BMS is the BRIDGIT bridge management system [3]. A bridge in a BMS is represented by structural elements defined as a set of common bridge components. For each bridge, the conditions of these elements are assessed visually during periodic inspections, and reported into the BMS. The condition data at each inspection consist of the total quantity of the element divided through a number of condition states; from the 'as good as new' condition to the most severe state of deterioration. This is a common practice for representing infrastructure condition data, starting with the discrete condition rating scale from 0 to 9 adopted by the U.S. Federal Highway Administration [4], and followed by the Pontis condition rating scale, and that of most other bridge inspection procedures. This process simplifies the inspections, but more importantly the modeling of the element/bridge/network condition and the maintenance optimization. The condition assessment and prediction is done by the application of a Markov chain model. The Markov chain model can be found in the maintenance and repair problems since the early 60's [5]. It is introduced to the maintenance of road infrastructure by Golabi et al. (1982) [6], and is made to drive the Pontis bridge management system. In the Markov model, the condition of a bridge element takes discrete states and the transitions from one state to the other are modelled with a Markov chain. While this approach has become standard, there have been a number of criticisms associated with it. In this article, we discuss the fundamentals and argue for the applicability of the gamma process and other stochastic processes for modelling structural deterioration.

\section{THE MARKOV CHAIN MODEL}

Markov model formulations are appealing to manage infrastructure because they provide a framework that accounts for the uncertainty and the optimal policies can be obtained by solving simple programming problems. A number of criticism points have been made against the usefulness of the model [1]; (i) bridge element performance is not addressed from a reliability viewpoint, (ii) the Markovian assumption does not take into account the history of the bridge deterioration, and (iii) bridge system performance is not generally addressed. Among these limitations, a most important one is the inability of the Markov model, by assumption, to capture the time effect of deterioration. It is clear to many practitioners that the deterioration rate tends generally to increase with time, and amount of deterioration. This observation conflicts with the stationarity assumption of the Markov model where the transition probabilities do not change with time. In the context of the estimation of the Markov model transition probabilities, Madanat et al. (1995) [7] make a number of observations. They point to the fact that the methods used in estimating these probabilities are ad-hoc and suffer from important methodological limitations; (i) the change in condition from one inspection to the next is not modelled explicitly, failing to capture the structure of the deterioration process, (ii) consequently, 
the model fails to capture the inherent non-stationarity of the deterioration process, and (iii) the approach does not recognize the latent nature of deterioration. Since deterioration is an unobservable process, it is not the state of the observable condition that should be modelled, but rather the process that generates these conditions.

\section{Transition Probabilities}

A practical difficulty with the estimation of the transition probabilities is the lack of data for some condition states. The severe conditions of elements of a bridge are rarely observed, due to the maintenance effort. Only the first states transition probabilities are estimated properly. Fig. 1 shows the frequency of units of different elements in the possible condition states, when inspected. An element on a bridge is measured in a number of units, either in square meters $\left(\mathrm{m}^{2}\right)$ if it is a surface, in meters $(m)$ for some elements such as railing and joints, or units (ea) for timber elements. The examples of Fig. 1 are taken from a network of bridges in the study we mention in the next section. They show that most of the element units are in state 2 when they are not in the 'as good as new' or 'full condition' state 1. While it depends on the element and on the budget, many elements are brought back to the full condition state from state 2 . This implies that little data are available about transitions to more severe conditions. Pontis supplements the data with expert knowledge at the beginning of system implementation due to the scarcity of data at that stage. As more data become available, the probabilities are to be updated using a Bayesian method. This is a major shortfall, as many managers are reticent about the use of subjective assessment, particularly if there is little chance that enough data will appear to adjust the subjective input.

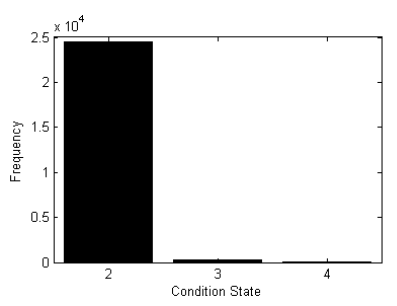

a)

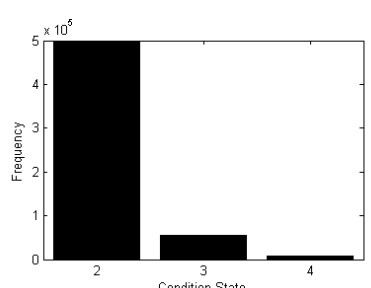

b)
Fig. 1 Condition frequency for a) Example 1, b) Example 2

\section{Stationarity Assumption}

The theoretical weakness of the Markov model is the assumption of stationarity. The model assumes that the time of transition from one state to another is distributed exponentially. This assumption allows the application of the Markov model by which an old unit of element and a newer one are equally likely to move to the next condition [2]. This is a rigid structure imposed on the deterioration model. This weakness is acknowledged in most studies, and yet the Markov model has been adopted from the beginning and remains in most bridge management systems. It provides a solution for the estimation of future conditions and prescribes how to invest maintenance funding. A predictive solution relies on the validity of the underlying deterioration model. Scherer and Glagola (1994) [8], Wirahadikusumah et al. (2001) [9] and Morcous (2006) [10], among others have studied the validity of the state independence assumption of the Markov model. This assumption assumes that the future condition of a bridge element depends only on its present condition and not on its past condition, implying that bridge deterioration is a stationary process. While the simplifying assumption holds in some studies, it is not substantiated with a lot of evidence, the available condition data not providing adequate information about all possible condition transitions.

\section{THE RTA LEVEL-2 INSPECTION DATA}

A study is conducted for the Roads and Traffic Authority of the state of New South Wales. The goal in the case study is to develop deterioration models for the assessment of future conditions of elements/bridges/networks of bridges. The data provided consist of inspection records conducted periodically on all bridges in the state. The inspections are visual for most elements and the condition states are discrete. An element consists of a number of quantity units. Each unit is judged to be in one of $n$ states, $n=3,4$ or 5 , depending on the element type. Condition state 1 represents the 'as good as new' condition, no-deterioration state, while condition states $2, \ldots, n$ mark increasing levels of deterioration. There are 4,945 structures and 66 elements considered in the study, for which over 230,000 inspection records exist. The records go back 15 years, and are up to the present time. Among the recorded entries is $q$, the total quantity of the element, $q_{1}$ the quantity in condition state 1 on the day of that inspection for that particular element, $q_{2}$ the quantity in state 2 , to $q_{n}$ the quantity in condition state $\mathrm{n}$. The inspected quantities for each element are measured either in square meters $\left(m^{2}\right)$ if it is a surface, in meters $(m)$ for some elements such as railing and joints, or units (ea) for timber elements. The structures have on average about 10 elements, with more than 30 in some cases. Each element was inspected approximately every two years. The elements are concrete elements, steel elements with lead based paint, steel elements with other protective treatment, timber elements, joints, bearings, railings and others. Some of the more common types are concrete pre-tensioned girder, concrete reinforced pre-stressed pile, concrete deck slab, concrete culverts, steel rolled beams / I girders with lead based paint protective coating, timber beam / cross girder, pourable / cork joint seal, 
elastomeric bearing, metal bridge railing and masonry / brick / reinforced earth.

\section{Condition Index}

Deterioration in elements of bridges is best measured as a continuous variable representing for example the percentage of degradation. Given the large surfaces and number of items to be inspected, it is hard to measure visually percentages. The condition data at each inspection therefore consists of the total quantity $q$ of the element being divided through judgment into $n$ states. $q=\sum_{i=1}^{n} q_{i}$ is satisfied in all inspection records. The percentage of undamaged quantity is $q_{1} / q \times 100 \%$. The corresponding proportion of deteriorated element is $q_{d}=\sum_{i=2}^{n} q_{i} / q, q_{d}=1-q_{1} / q$. If one was to ignore the degree of deterioration and consider only the proportion of damaged quantity, then $C=q_{1} / q \times 100$ provides a first level of information on the condition of the element. In our study, the condition data $q_{1} \ldots q_{n}$ of an element is converted to an univariate measure $C$, using the notion of 'Condition Index'. A number of condition indices were formulated. These indices can be related to the California bridge health index [11], a ranking system that takes values in $[0,100]$. The California Department of Transportation was involved in the development and implementation of Pontis. A condition index has two functions; (i) its use in a cost / benefit analysis where the condition history of a structure can be estimated with the inspection data of its elements, through the use of a weighted sum of the conditions of the elements, and (ii) its use in the study of deterioration by modelling the univariate measure in time.

\section{THE DETERIORATION RATE}

In turning the vector of quantities $q_{1} \ldots q_{n}$ into a single value $C_{\text {elem }}(t)$ where $t$ is time, the dimensionality of the problem is reduced and one can apply mathematically tractable models. This univariate quantity can be studied over time using a stochastic process. The element condition is a value between 0 and $100 \%, 100 \%$ being the 'as good as new' condition (no deterioration) state. An element can be in one of 11 states at an inspection time. The 11 states are mutually exclusive and exhaustive events, defined using two consecutive element conditions. Some of these states are $\left(S_{1}\right)$ : the element condition $C$ is $100 \%$ and $100 \%$ at the previous inspection, $\left(S_{2}\right)$ : the element condition $C$ is less than $100 \%$, but positive, while $100 \%$ at the previous inspection, $\left(S_{3}\right)$ : the element condition $C$ is $0 \%$ while $100 \%$ at the previous inspection, $\left(S_{7}\right)$ : the element condition $C$ is less than $100 \%$, but positive, and less than at the previous inspection where it was less than $100 \%$, but positive, and $\left(S_{8}\right)$ : the element condition $C$ is $0 \%$, while it was less than $100 \%$, but positive at the previous inspection. These states are of relevance when analyzing the deterioration. The other states are due to maintenance, when a renovation or repair improves the condition of the element or brings it back to 'as good as new'.

\section{Deterioration Measure}

The deterioration is defined as a function of the condition, $Z=100-C,\left[C \equiv C_{\text {elem }}(t)\right] . Z_{1}, Z_{2}, Z_{3}, Z_{7}$ and $Z_{8}$ are the deterioration quantities corresponding to the states $S_{1}, S_{2}, S_{3}, S_{7}$ and $S_{8}$. These variables are of relevance when studying the deterioration process, along with their inter-inspection times. The time at which a deterioration increase, $d Z$ in a time interval $d t$, occurs is important. The pairs $(d t, d Z)$, for $d Z>0$, and the time at which $d t$ starts are information used to estimate deterioration.

\section{Time Pattern}

In order to study the distribution of the deterioration random variables $Z_{2}$ and $d Z_{7}$ (the increase associated with $Z_{7}$ ), we need data that occur at the same time. In practice, inspections are not always strictly periodic. However, the inter-inspection times can be adjusted so that they are grouped together, making it possible to study the probabilistic behavior of deterioration as a random variable at different times. The adjustment was made through rounding off the inspection times. The pattern showed that the inspection times were meant to be 2 years apart. Figure 2 shows the conditions $C=100-Z_{2}$ plotted in dots, of some concrete element type chosen as an example, as they occur in time from a renewal, on the structures the element belongs to. The stars are the adjusted conditions. 


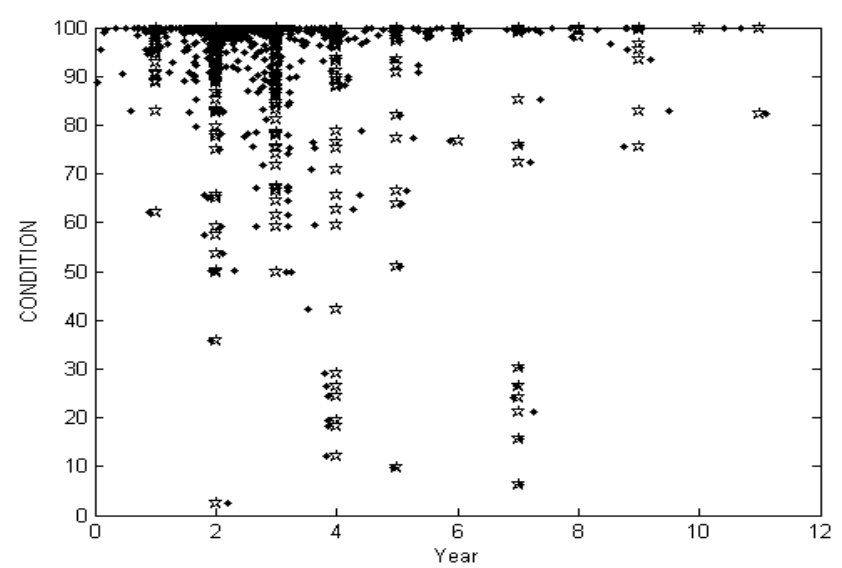

Fig. 2 Condition data of a concrete element type

Judgment was used in eliminating data for inspection time intervals that exceed 4 years. Beyond 4 years, the data start thinning. In the case of this element, from the original 913 conditions, $C=100-Z_{2}, 836$ data points adjusted in time are plotted in Fig. 3. Next, we used a least squares approach in fitting the data to a nonlinear deterioration curve $100-\mu t^{q}$, with parameters $\mu$ and $q$. Fig. 3 displays the least squares results using (i) the condition means at times 1,2,3 and 4 years, (ii) all the adjusted data, and (iii) the actual data. $q$ was found to be $2.7,2.4$ and 2.4 , respectively, and $\mu=0.29,0.404$, 0.408 , showing that the adjustment to the data was minimal. The time pattern of the deterioration of the element fits the behaviour of a stochastic process with mean function $\mu t^{q}$.

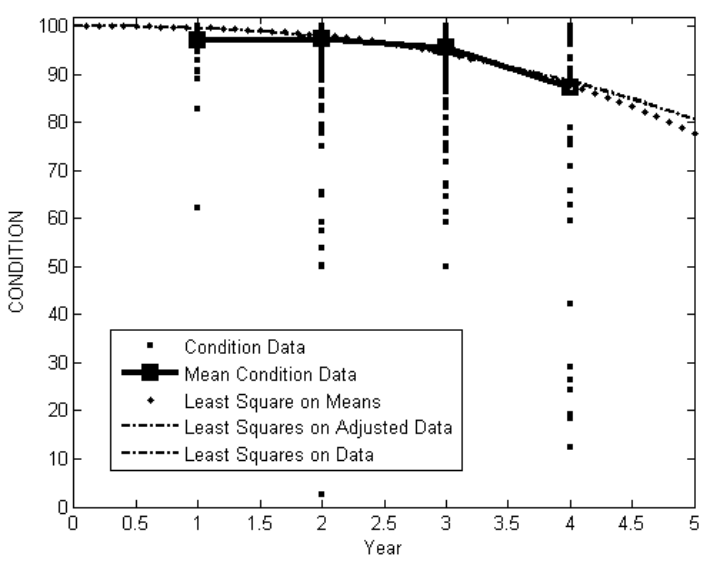

Fig. 3 Condition of a concrete element

\section{Probability Distribution Fit}

The distributions of $Z_{2}$ at different points in time characterize the stochastic process. In the case of most of our elements, the probability distribution showed a good fit to the gamma distribution (Fig. 4). This agrees with the properties of the gamma deterioration process, where the distributions of incremental deteriorations are gamma distributed [12]. This was observed for the concrete and steel elements, as well as railing and joints and some timber elements. The lognormal distribution was also found to be a good fit (Fig. 5).

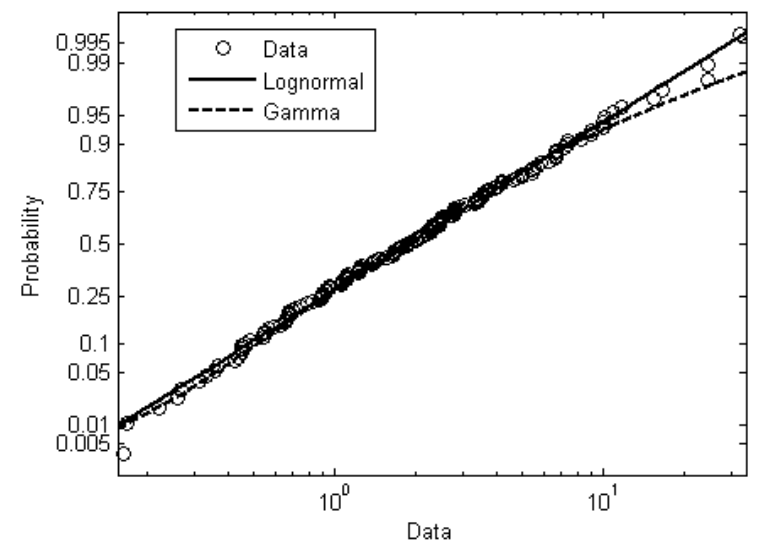

Fig. 4 Gamma and Lognormal probability distribution fits

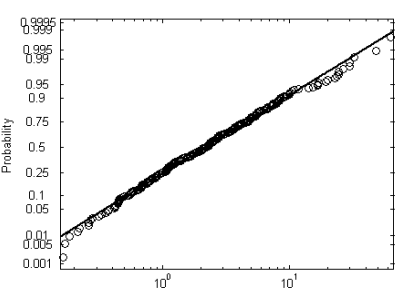

a)

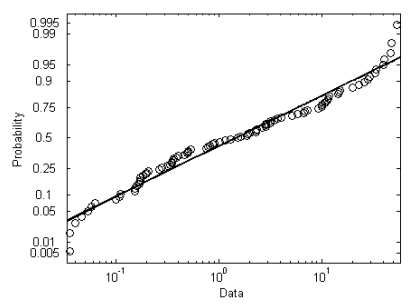

b)
Fig. 5 Lognormal fit for a) Concrete, b) Steel element

In the gamma process, not only is the distribution a gamma distribution when measured from the start of time, but any incremental distribution is also a gamma distribution. That is, if taking an interval in time and measuring the difference, increase, in the quantity of interest, the distribution of such an increase is also a gamma distribution. In addition, the increases in nonoverlapping time intervals are independent. This makes for a number of assumptions to be checked. Fig. 6 shows the distribution fit to the gamma and lognormal probability distributions for the increase in deterioration $d Z_{7}$, in a time interval $d t=2$ years, when recorded at $t+d t$ years after a renewal. The plots in Fig 6 are for $t=0,1,2, \ldots, 11$ left to right, top to bottom. 

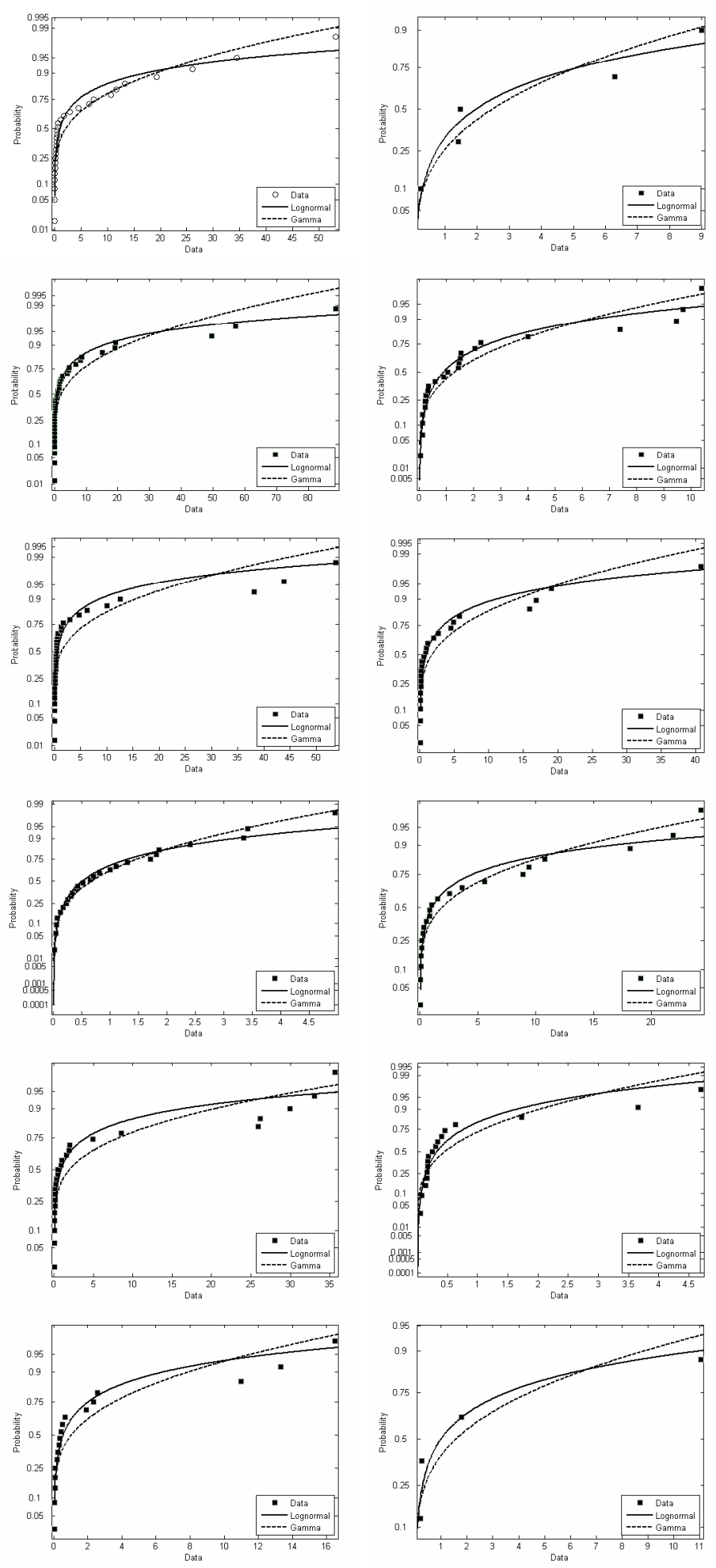

Fig. 6 Probability plots for deterioration increase

This example is taken for a concrete element common on a number of structures so that the data on $d Z_{7}$ allow a conclusive distributional fit. The good fit was also observed within classes of structures, following stratification of the data. We observed that whenever $d Z_{7}$ data are abundant enough, the stochastic process assumptions can be accepted. The data of the case study show the behavior of a stochastic process with selected probability distributions.

\section{Gamma Process Application}

For the purpose of building a statistical model, these observations promote the use of the gamma process with mean deterioration function $\mu t^{q}$. This process mean function includes the stationarity case for $q=1$. The gamma process is defined by van Noortwijk (2009) [12] in the context of the deterioration of structures. To illustrate the estimation procedure, we apply it to a concrete element and observe a rate $\hat{q}=3.3$ (Fig. 7).

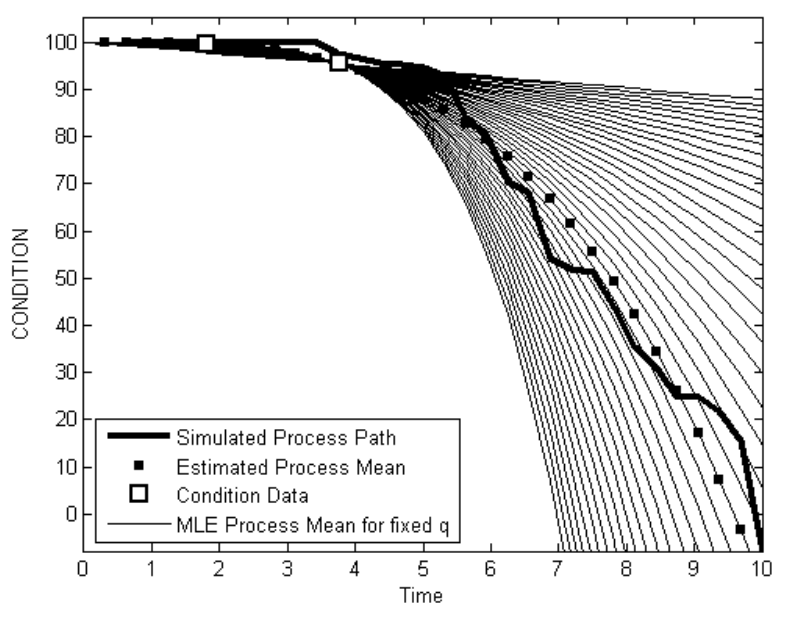

Fig. 7 Gamma process estimation

Two data points are used; $(15.81,99.69)$ and $(17.75$, 95.46) with the renewal at year 13.92 since the start of the database. This example was chosen for illustrative purposes. In many cases, the bridge elements showed lower rates.

\section{THE GAMMA PROCESS}

The gamma process can capture the temporal variability of degradation. The argument is made in a series of papers by Pandey and van Noortwijk (2004) [13], van Noortwijk et al. (2005) [14] and Pandey et al. (2007) [15]. The gamma process can be found in its modern application to structures in the late 90's by van Noortwijk (1998) [16] and van Noortwijk and Klatter (1999) [17]. The idea of the use of a gamma process can be found earlier in the Netherlands where generalized gamma processes were used to model decision problems for optimizing maintenance of the sea-bed protection of the Eastern-Scheldt barrier, berm breakwaters, and dykes [18]. Empirical studies showed that the expected deterioration in some cases followed the power law $a t^{b}$, where $t$ is the time. This function of time is incorporated into the gamma process and used to model structural 
deterioration. The advantage of the gamma process is recognized and applied in many structural studies [19][21]. van Noortwijk (2009) [12] provides a comprehensive overview of the use of the gamma process in the maintenance of structures.

\section{Definition}

In the context of structural deterioration, the gamma process is defined as follows: Let $v(t)$ be a nondecreasing, right continuous, real-valued function for $t>0$, with $v(0)=0$. The gamma process with shape function $v(t)>0$ and scale parameter $u>0$ is a continuous-time stochastic process $Z(t), t>0$ with the following properties;

$Z(0)=0$ with probability 1 ,

$Z(\tau)-Z(t) \sim G(v(\tau)-v(t), u)$ and

$Z(t)$ has independent increments,

where $G(z \mid v, u)=u^{v} z^{v-1} e^{-u z} / \Gamma(v)$ is the gamma probability density function defined for $z>0$. The process can be parameterized. Letting $v(t)=\mu^{2} t^{q} / \sigma^{2}$ and $u=\mu / \sigma^{2}$, the mean and variance of the deterioration $Z(t)$ are:

$$
\begin{aligned}
& E(Z(t))=\mu t^{q} \\
& V(Z(t))=\sigma^{2} t^{q}
\end{aligned}
$$

Given a set of observations of the deterioration process $Z(t),\left\{z_{i}\right\}_{i=1}^{n}$ for times $\left\{t_{i}\right\}_{i=1}^{n}$, the maximization of the likelihood function provides estimates of the three parameters $(\mu, \sigma, q)$. This involves the search, $q$ fixed, for the zero of a function, where $\hat{\sigma}$ is solution of

$$
\sum_{i=1}^{n} w_{i}\left\{\psi\left(\frac{\hat{\mu}^{2}}{\sigma^{2}} w_{i}\right)-\log \delta_{i}\right\}=t_{n}^{q} \log \left(\frac{z_{n}}{t_{n}^{q} \sigma^{2}}\right)
$$

where $\delta_{i}=z_{i}-z_{i-1}$ and $\quad w_{i}=t_{i}^{q}-t_{i-1}^{q}, \quad i=1, \ldots, n$, $z_{0}=0$ with $t_{0}=0$, and $\hat{\mu}=z_{n} / t_{n}^{q} \cdot \psi$ is the Digamma function.

\section{Simulation and Estimation}

We experimented with the process using simulation and estimating the parameters with the maximum likelihood approach. With few data points, the model captures the deterioration process efficiently. The lower the data points are along the curve, the better the estimation. To illustrate, we assume that three data points are observed; $\left(y_{1}, c_{1}\right)=(2,99.5),\left(y_{2}, c_{2}\right)=(4,99.4)$ and $\left(y_{3}, c_{3}\right)=(6$, 95.2). This means that some years after the start of the database, at a renewal point, the element deterioration is $z_{0}=0$, time is reset to $t_{0}=0$ and the condition $C_{0}$ is set to $100(\%)$. At the next inspection, 2 years later, $\left(y_{1}, c_{1}\right)$ is recorded leading to $\left(t_{1}, z_{1}\right)=\left(2,100-c_{1}\right)=(2$, $0.5)$. Similarly, $\left(t_{2}, z_{2}\right)$ and $\left(t_{3}, z_{3}\right)$ are obtained. The first deterioration is of $0.5 \%$ magnitude, then $0.1 \%$. Then a further $4.2 \%$ of the element deteriorates. Varying the deterioration rate of the process, $q=1, \ldots, 5, \mu$ and $\sigma$ are estimated using the maximum likelihood method for each $q$ value. The resulting process mean functions, $100-\hat{\mu} t^{q}$, for corresponding $q$, are plotted in Fig. 8. $\hat{\mu}$ is the maximum likelihood estimate of $\mu$ for given $q$. Applying the maximum likelihood principle again, the deterioration rate is estimated to be $\hat{q}=2$, for corresponding $\hat{\mu}=0.1333$ and $\hat{\sigma}=0.4875$. The estimated condition process mean function $100-\hat{\mu} t^{\hat{q}}$ is shown as a dotted line in Fig. 8.

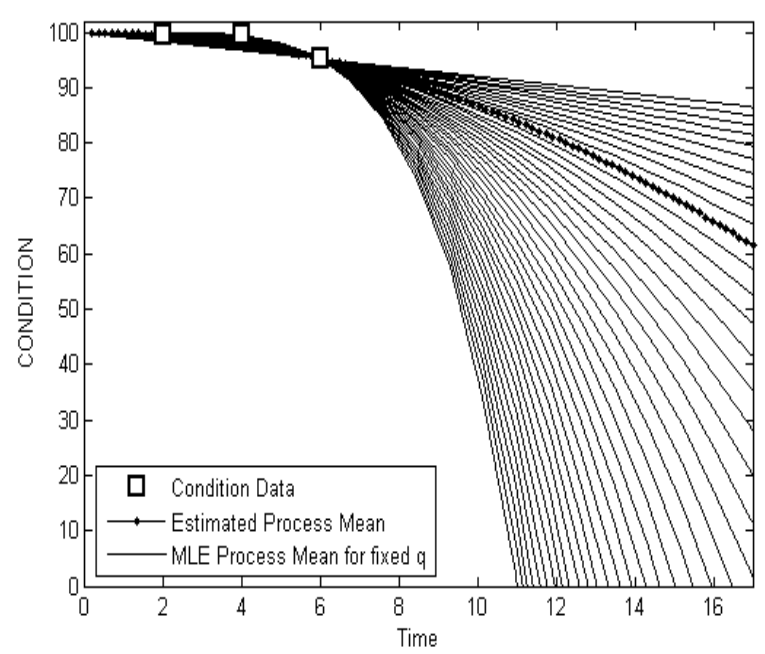

Fig. 8 Maximum likelihood estimation

Using the estimated parameters $(\hat{\mu}, \hat{\sigma}, \hat{q})$, a condition path is simulated for illustrative purposes and shown in Fig. 9. 


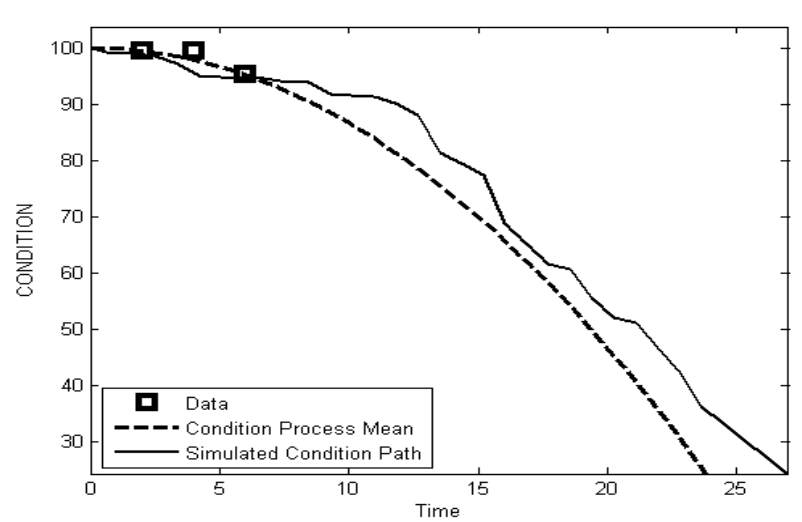

Fig. 9 Simulated condition path

Replicating 50 times, the condition data are shown in Fig. 10. The data points are not connected with lines, to better illustrate the behaviour of the stochastic process at a fixed point in time. For a given point in time, the corresponding data are gamma distributed.

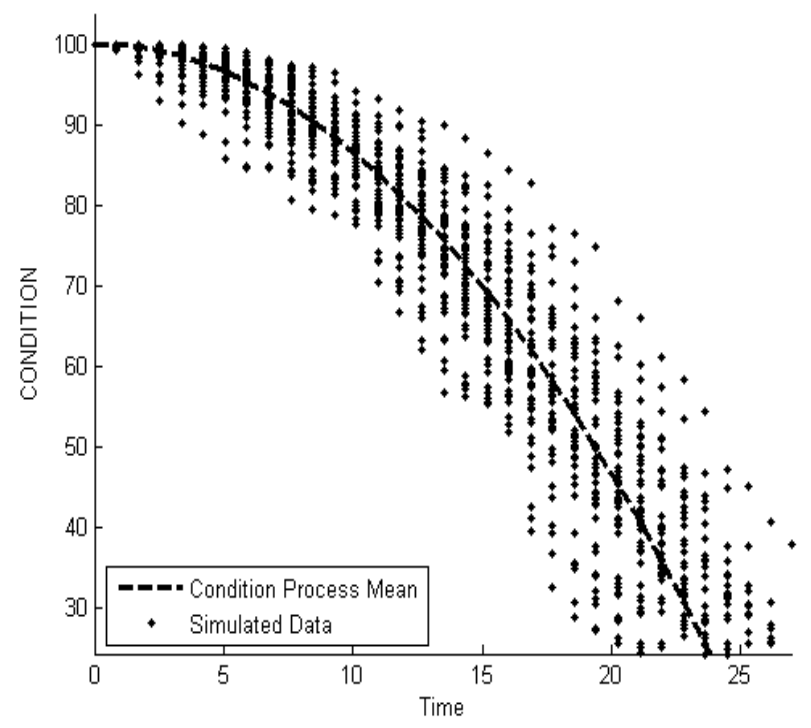

Fig. 10 Simulated condition paths for 50 replications

Fig. 11 shows the theoretical mean deterioration process of a gamma process, along with the ensuing condition mean process. The theoretical distributions of the accumulated deterioration $\left(Z_{7}\right)$ and the increase deterioration $\left(d Z_{7}\right)$ are schematized.

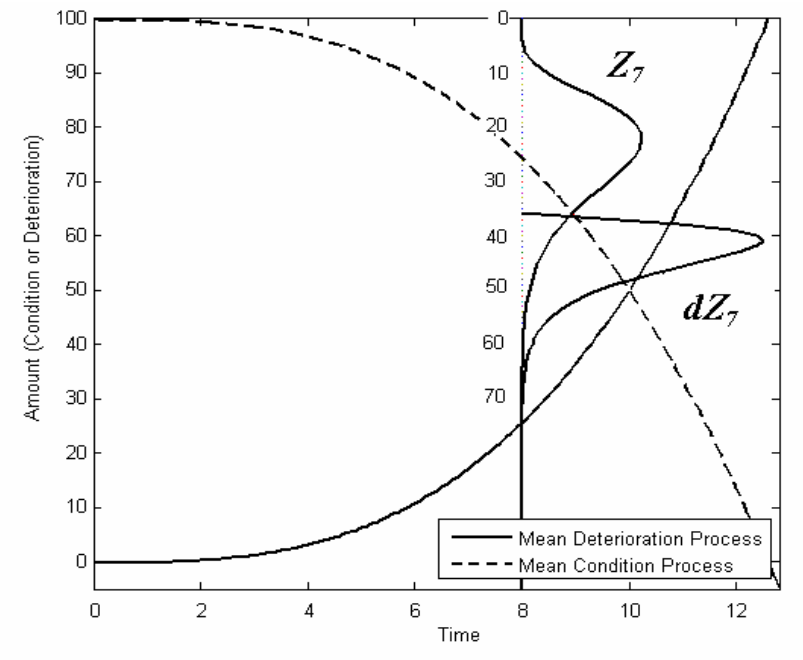

Fig. 11 Theoretical mean deterioration and condition

\section{Accuracy of the Estimation Procedure}

In experimenting with the gamma process, we observe that with few data points the model captures the deterioration process efficiently. The lower the data points are along the curve, the better the estimation. This is a significant observation. The high deterioration points for an element of a bridge are not many and the few that are observed do not provide good estimates for the Markov transitions to high deterioration states. However, few of these data points provide a good estimation when using a gamma process model. To illustrate this point, we choose to simulate 50 condition paths using the same estimated parameters, whose theoretical process mean is shown by the dashed line in Fig. 12. For each simulated path, three data points are taken, using the times $t_{1}=1.6875, t_{2}=5.0625$ and $t_{3}=10.9688$. For each path, the data may differ, and are plotted as small squares in the figure. The resulting MLE estimated condition process means are shown in Fig. 12. Their average is plotted as a line with small circles.

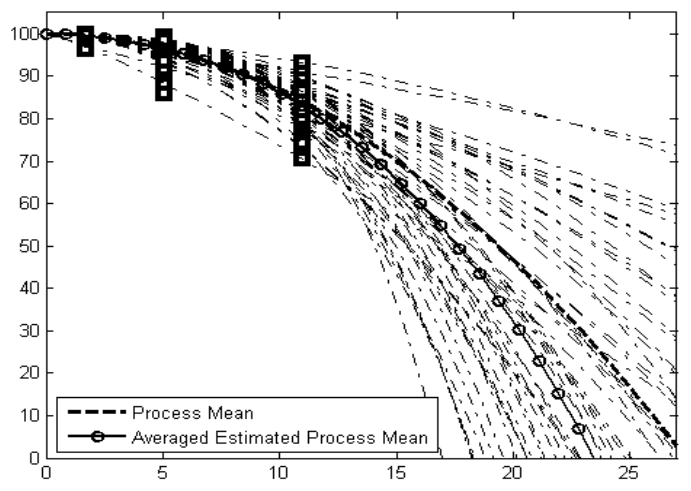

Fig. 12 Averaged estimated process means 
It is clear that the estimation is more efficient at the top of the curve where the data lie. This can be verified theoretically for a fixed $q$, since the estimate of $\hat{\mu}$ depends on the last data point $\left(t_{n}, z_{n}\right)$. However, in estimating the triplet $(q, \mu, \sigma)$, a closed form solution is not available. We observe empirically that the lower the condition data is on the curve, that is the higher the observed deterioration, the better the estimation of the condition (deterioration) process. Keeping $t_{1}=1.6875$ and $t_{2}=5.0625$ but varying $t_{3}$, we observe the average estimated process mean performs better as the third data point goes deeper in time, and hence in deterioration (see Fig. 13). At $t_{3}=22.78$, the deterioration is $69.2 \%$, for a corresponding condition of $30.8 \%$. This leads, on average, to a perfect estimation of the process mean curve. This point is further illustrated in Fig. 14 where we choose $t_{3}=24.4688$, for a corresponding $80 \%$ theoretical deterioration. Fig. 14 shows the corresponding statistics of the 50 simulation-MLE results.

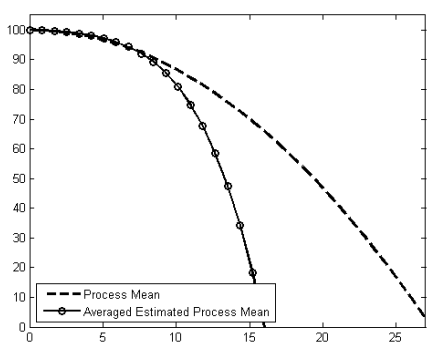

a)

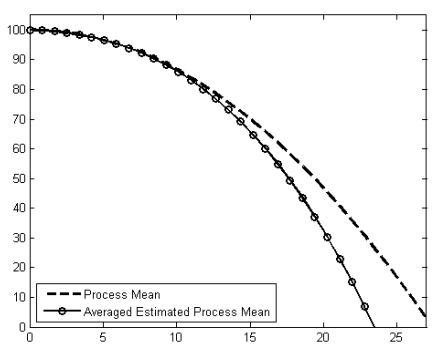

b)

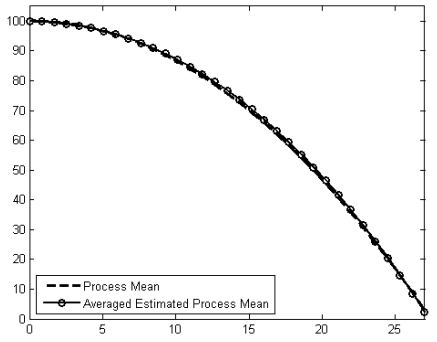

c)

Fig. 13 Average estimated process mean for a) $t_{3}=6.75$, b) $t_{3}=10.96$, c) $t_{3}=22.78$

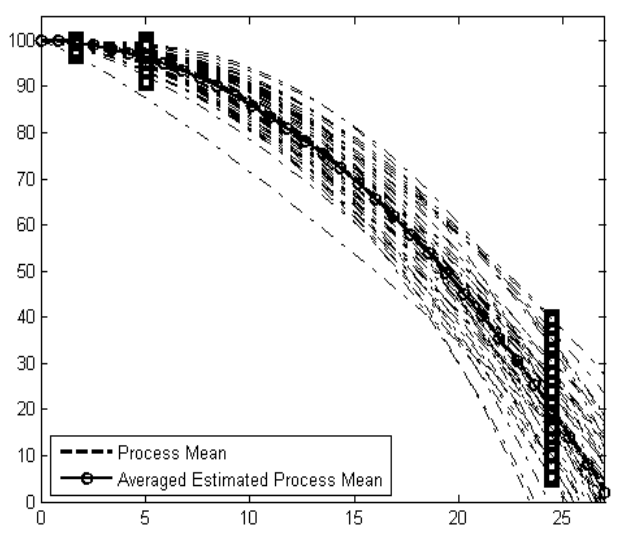

a)

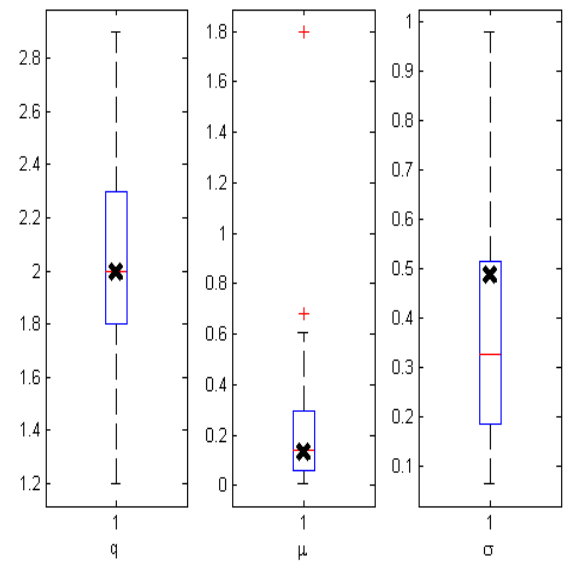

b)

Fig. 14 Average estimated process mean with deep information a) Averaged MLE mean process, b) MLE statistics ( $x=$ Parameter value)

For an element whose deterioration behaves in time according to a gamma process, any information on the condition path leads to a better estimation. But the deeper that information is along the curve, the higher the observed deterioration, the better the theoretical process mean is estimated. The importance of such estimation is apparent in the execution of the final exercise, the maintenance optimization. In estimating at what time the condition of an element will reach a target level, and the ensuing economic consequences of corresponding maintenance times, the accuracy is of significant importance. It is illustrated in Fig. 15, where two different condition paths lead to two very different times, $t_{1}^{*}$ and $t_{2}^{*}$, to reach the $20 \%$ deterioration target. There is approximately a 2 years difference between the two maintenance times, which can be of significant difference in terms of cost if applied to a large number of elements on different bridges or to expensive elements. 


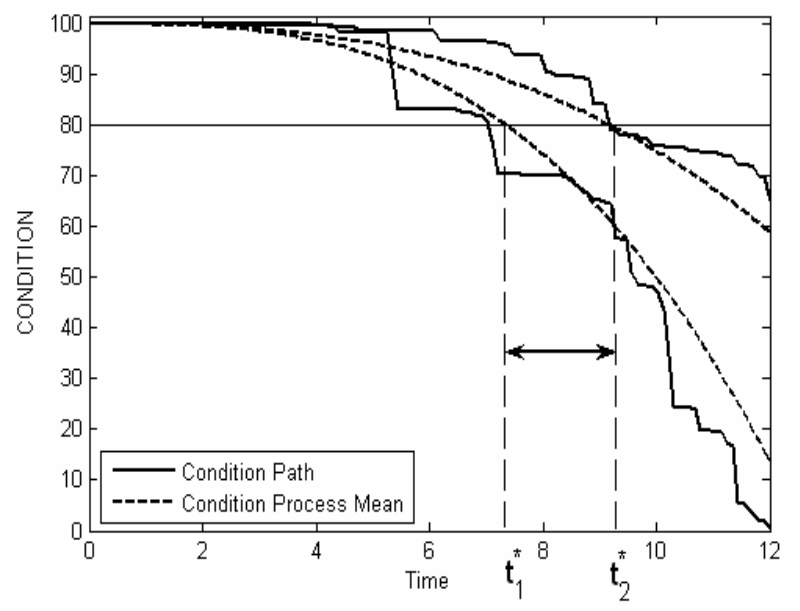

Fig. 15 Time to reach target level

\section{Aggregating Data}

The exercise of estimating the process mean curve is at the heart of the maintenance optimization problem. While the decision model requires the declaration of costs, a non trivial part, estimating properly the condition curve is the essential part of the problem. The data for each individual bridge aren't enough to estimate the parameters $\mu, \sigma$ and $q$ properly. Often, the path $\left\{z_{i}\right\}_{i=1}^{n}$ for times $\left\{t_{i}\right\}_{i=1}^{n}$ does not extend beyond $\mathrm{n}=1$ or 2 , before the element is brought back to the full condition state $Z=0$. One way around this problem is to aggregate the data by dividing the bridges into similarity classes. Bridges are grouped through the identification of major elements. Then within the classes of similar bridges, a second stratification occurs according to influencing factors; traffic load, age, region and environmental stress. The estimation of the parameters within the class structures results in an effective deterioration assessment and prediction. The approach of aggregating data is the one taken in most scenarios. Regardless of the statistical model, be it the Markov chain or a stochastic process such as the gamma process, one is often forced to pull information about an element type from different bridges and estimate and predict the deterioration. But in the gamma process, the approach provides a more efficient estimation procedure. While few data points for high deterioration provide at best weak estimates for the transition probabilities to the corresponding states in the Markov model, in the gamma process, they help pin down the deterioration curve more accurately, as seen in the simulation analysis.

\section{First Observation Data}

In the special case where only the first deteriorations, observed at the first inspection after a renewal, are available, the solution is similar to the one for a single path seen above, but the search for $\hat{\sigma}$, for fixed $q$, involves the equation

$$
\sum_{i=1}^{m} w_{i}\left\{\psi\left(\frac{\hat{\mu}^{2}}{\sigma^{2}} w_{i}\right)-\log \delta_{i}\right\}=\left(\sum_{i=1}^{m} w_{i}\right) \log \left(\frac{\hat{\mu}}{\sigma^{2}}\right)
$$

where $z_{i}$ is the deterioration of the $i^{\text {th }}$ element in the class of structures, $\delta_{i}=z_{i}$ and $w_{i}=t_{i}^{q}, i=1, \ldots, m$ and $\hat{\mu}=\sum_{i=1}^{m} z_{i} / \sum_{i=1}^{m} t_{i}^{q}$. In general, due to the independent increments property and with the assumption of independence between elements on different structures, the likelihood function can be written and maximized to estimate the parameters $\mu, \sigma$ and $q$ [21].

\section{LOGNORMAL DIFFUSION PROCESS}

Another candidate for the modelling of the deterioration is the lognormal diffusion process. Used in statistical modelling, it has been mainly confined to the analysis of economic data [22]. The lognormal diffusion process with exogenous factors is defined by the process $\left\{X(t), t \in\left(t_{0}, T\right], t_{0} \in \mathfrak{R}\right\}$ with positive values and infinitesimal moments:

$$
\begin{aligned}
& A_{1}(x, t)=x h(t) \\
& A_{2}(x, t)=\sigma^{2} x^{2}
\end{aligned}
$$

where $h(t)=\beta_{0}+\sum_{j=1}^{q} \beta_{j} F_{j}(t), \beta_{j} \in \mathfrak{R}, \sigma>0$ and $F_{j}$ are time-continuous functions in $\left(t_{0}, T\right] j=1, \ldots, q$. The transition probability density function of this process is a lognormal distribution and the trend is given by the expression,

$$
E(X(t))=E\left(X\left(t_{0}\right)\right) \exp \left(\int_{t_{0}}^{t} h(\tau) d \tau\right)
$$

The estimation of the parameters $\left(\beta_{0}, \ldots, \beta_{q}, \sigma\right)$ is conducted through the maximization of likelihood as for the Gamma process. Replacing the process mean $\mu t^{q}$ of the gamma process with a trend $e^{\lambda t^{q}}-1$ in the lognormal diffusion process can capture the deterioration process. 


\section{CONCLUSION}

Structural deterioration assessment and condition prediction of bridges is a subject of interest that has far reaching consequences, both in terms of public safety and budgeting. This article discusses the theoretical foundations of the model most used for assessing structural deterioration in bridge elements. A modern view is proposed using the gamma process that has proved successful in structural deterioration assessment. Experimentation with the process is shown using simulation and estimation of the parameters of the model. With few data points, the model captures the deterioration process efficiently. In the case study, a probability distribution fit is observed that supports the use of the gamma process as well as leads to the consideration of other stochastic deterioration processes.

\section{REFERENCES}

[1] D.M. Frangopol, J.S. Kong and E.S. Gharaibeh, "Reliabilitybased life-cycle management of highway bridges", Journal of Computing in Civil Engineering, vol. 15, no. 1, pp. 27-34, Jan. 2001.

[2] K. Golabi and R. Shepard, "Pontis: A system for maintenance optimization and improvement of US bridge network", Interfaces, vol. 27, no. 1, pp. 71-88, Jan.-Feb. 1997.

[3] H. Hawk and E.P. Small, "The BRIDGIT bridge management system", Structural Engineering International, vol. 8, no. 4, pp. 309-314, Nov. 1998.

[4] Federal Highway Administration, Recording and coding guide for structure inventory and appraisal of the nation's bridges, U.S. Department of Transportation, Washington, D.C., U.S.A, 1979.

[5] M. Klein, "Inspection-maintenance-replacement schedules under Markovian deterioration", Management Science, vol. 9, no. 1, pp. 25-32, Oct. 1962.

[6] K. Golabi, R.B. Kulkarni and G.B. Way, "A Statewide Pavement Management System", Interfaces, vol. 12, no. 6, pp. 5-21, Dec. 1982.

[7] S. Madanat, Mishalani, R. and W.H. Wan Ibrahim, "Estimation of infrastructure transition probabilities from condition rating data", Journal of Infrastructure Systems, vol. 1, no. 2, pp. 120-125, June 1995.
[8] W.T Scherer and D.M. Glagola, "Markovian models for bridge maintenance management", Journal of Transportation Engineering, vol. 120, no. 1, pp. 37-51, 1994.

[9] R.Wirahadikusumah, D. Abraham and T. Iseley, "Challenging issues in modeling deterioration of combined sewers", Journal of Infrastructure Systems, vol. 7, no. 2, pp. 7784, June 2001.

[10] G. Morcous, "Performance Prediction of Bridge Deck Systems Using Markov Chains", Journal of Performance of Constructed Facilities, vol. 20, no. 2, pp. 146- 155, May 2006.

[11] R.W. Shepard and M.B. Johnson, California Bridge Health Index, IBMC-005, California Department of Transportation, International Bridge Management Conference, Denver, Colorado, Preprints, Volume II, K1, 1999.

[12] J.M. van Noortwijk, "A survey of the application of gamma processes in maintenance", Reliability Engineering and System Safety, vol. 94, pp. 2-21, 2009. Available online March 2007.

[13] M.D. Pandey and J.M. van Noortwijk, "Gamma process model for time-dependent structural reliability analysis", In Bridge Maintenance, Safety, Management and Cost, E. Watanabe, D.M. Frangopol, and T. Utsonomiya, editors, Proceedings of the Second International Conference on Bridge Maintenance, Safety and Management (IABMAS), Kyoto, Japan, 18-22 October 2004. London: Taylor \& Francis Group.

[14] J.M. van Noortwijk, M.J. Kallen and M.D. Pandey, "Gamma processes for time-dependent reliability of structures", In Advances in Safety and Reliability, K. Kolowrocki, editor, Proceedings of ESREL 2005 - European Safety and Reliability Conference 2005, Tri City (Gdynia-Sopot-Gdansk), Poland, 2730 June 2005, pp. 1457-1464. London: Taylor\& Francis Group.

[15] M.D. Pandey, X.-X. Yuan and J.M. van Noortwijk, "The influence of temporal uncertainty of deterioration on life-cycle management of structures", Structure and Infrastructure Engineering, pp. 1-12, April 2007.

[16] J.M. van Noortwijk, "Optimal Replacement Decisions for Structures under Stochastic Deterioration", Proceedings of the Eighth IFIP WG 7.5 Working Conference on Reliability and Optimization of Structural Systems, Krakow, Poland, 1998, pp. 273-280, Ann Arbor: University of Michigan.

[17] J.M. van Noortwijk and H.E. Klatter, "Optimal inspection decisions for the block mats of the Eastern-Scheldt barrier", Reliability Engineering and System Safety, vol. 65, pp. 203-211, 1999. 
[18] J.M. van Noortwijk and P.H.A.J.M. van Gelder, "Optimal maintenance decisions for berm breakwaters", Structural Safety, vol. 18, no. 4, pp. 293-309, 1996.

[19] F.A. Buijs, J.W. Hall, J.M. van Noortwijk and P.B. Sayers, "Time-dependent reliability analysis of flood defences using gamma processes", In Safety and Reliability of Engineering Systems and Structures, Augusti G, Schueller GI, Ciampoli M, editors., pp. 2209-2216. Rotterdam: Millpress; 2005.

[20] J.M. van Noortwijk, J.A.M. van der Weide, M.J. Kallen, M.J. and M.D Pandey, "Gamma processes and peaks-over-threshold distributions for time-dependent reliability", Reliability Engineering and System Safety, vol. 92, pp. 1651-1658, 2007. Available online Dec. 2006.

[21] R.P. Nicolai, R. Dekker and J.M. van Noortwijk, "A comparison of models for measurable deterioration: An application to coatings on steel structures", Reliability Engineering and System Safety, vol. 92, pp. 1635- 1650, 2007. Available online Nov. 2006.

[22] R. Gutierrez, P. Roman, and F. Torres, "Inference on some parametric functions in the univariate lognormal diffusion process with exogenous factors", Test, vol. 10, no. 2, pp. 357373,2001

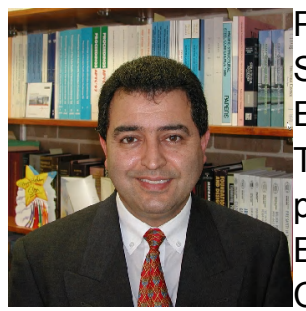

Professor Bijan Samali is Head of the School of Civil and Environmental Engineering at the University of Technology Sydney and has a personal chair in Structural Engineering. $\mathrm{He}$ is the Director of Centre for Built Infrastructure Research at the University of Technology Sydney. He has published over 300 technical papers in engineering journals and conference proceedings. His main research interests lie in the general area of structural dynamics including wind and earthquake engineering with special emphasis on structural control, dynamic measurement and analysis of buildings and bridges, damage detection and structural health monitoring, and use of smart materials in engineering applications. $\mathrm{He}$ has successfully supervised $8 \mathrm{PhD}$ students to date with another four conducting research under his supervision. $\mathrm{He}$ has over 24 years of academic experience and engagement with industry as a specialist consultant for over 25 years. Email: Bijan.Samali@uts.edu.au

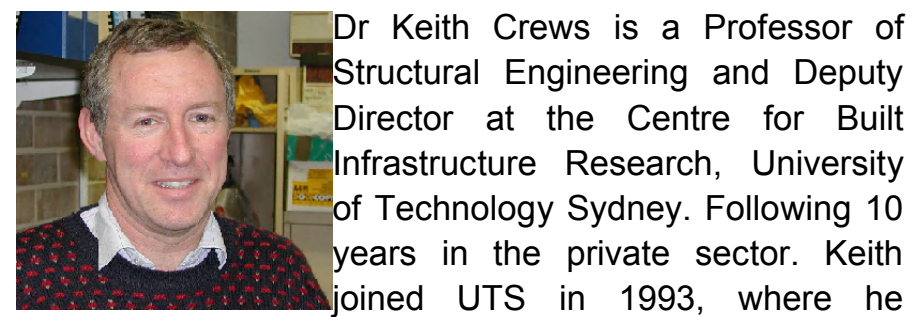

undertakes teaching, research and specialist consulting activities. He is an internationally recognized expert on timber structures, such as bridges, buildings and utility poles. He has published extensively, having authored over 300 papers and technical reports relating to the use, assessment, safety and reliability of structural timber. He is an active member of 4 SAA code committees, ISO TC165 (Timber Engineering) and CIB W18, as well as recently joining RILEM Committee TC 215. . Keith has received numerous awards for his research and is the recipient of the IEAust Structural College Eminent Speaker award for 2009. Email: Keith.Crews@uts.edu.au

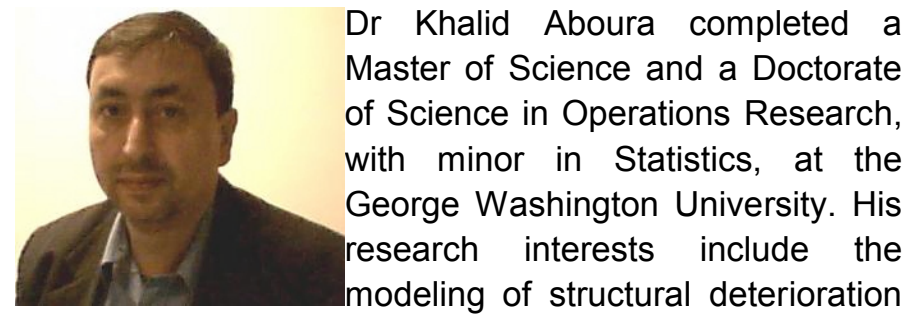
processes, the development of bridge management systems, probabilistic solutions in image analysis and the statistical foundations and the meaning of probability. Khalid Aboura has consulting experience and participated in research projects supported by government and research labs. He served as the Chairman of the Statistical Computing Section of the Washington Statistical Society. Email: Khalid.Aboura@uts.edu.au

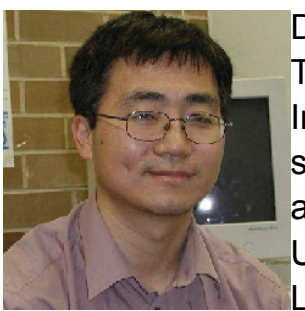

Dr $\mathrm{Li}$ received his $\mathrm{PhD}$ degree from Trinity College, University of Dublin, Ireland in 1993. He is currently a senior lecturer, Faculty of Engineering and Information Technology, University of Technology Sydney. Dr. i has twenty years experience in extensive research crossing different disciplines from aeronautical, mechanical to civil engineering. He has authored or co-authored 100 plus scholarly publications including a book and over 40 journal papers. His current research interests are in the areas of smart materials and smart structures, structural control for civil engineering, damage detection, structural health monitoring and structure rehabilitation using FRP.

Email: Jianchun.Li@uts.edu.au 